\title{
Numerical Field Calculation in Support of the Hardware Commissioning of the LHC
}

\author{
Nikolai Schwerg, Bernhard Auchmann, and Stephan Russenschuck
}

\begin{abstract}
The hardware commissioning of the Large Hadron Collider required the testing and the qualification of the cryogenic and vacuum system, as well as the electrical systems for the powering of more than 10000 superconducting magnets. Nonconformities had to be resolved within a tight schedule. In this paper, we focus on the role that electromagnetic-field computation has played during hardware commissioning in terms of the analysis of a magnet quench and electromagnetic-force calculations in busbars and splices, as well as field-quality prediction for the optimization of powering cycles.
\end{abstract}

Index Terms-Lorentz forces, quench calculation, superconducting magnets.

\section{INTRODUCTION}

W ITH THE Large Hadron Collider (LHC) [1], the particle-physics community aims at testing various fundamental theories by studying the collisions of counterrotating proton beams with a center-of-mass energy of $14 \mathrm{TeV}$. Physicists hope to prove the Higgs mechanism for generating elementary-particle masses of quarks, leptons, and the $\mathrm{W}$ and $\mathrm{Z}$ bosons. The LHC reuses civil-engineering infrastructure from the Large Electron-Positron Collider at the European Organization for Nuclear Research (CERN) that straddles the Swiss-French border near Geneva. The existing tunnel is $3.8 \mathrm{~m}$ wide and has a circumference of about $27 \mathrm{~km}$. With a given radius of the accelerator tunnel, the maximum achievable particle momentum is proportional to the operational field in the bending magnets. Superconducting dipole magnets cooled to $1.9 \mathrm{~K}$, with a nominal field of $8.33 \mathrm{~T}$, will allow energy values of up to $7 \mathrm{TeV}$ per proton beam [2].

During the design and the construction of the LHC, which was an undertaking of more than 20 years, various challenges had to be met in all domains of physics and engineering. Among these challenges are the following: material science for superconducting wires and cables; mechanical-engineering challenges for the construction of magnets weighing up to 20 ton, the physics of superfluid helium, and vacuum technology for insulation and beam vacuum; and electrical-engineering challenges for power supplies, current leads using high-temperature superconductors, superconducting busbars, diodes operating at cryogenic temperatures, and magnet-protection systems.

Manuscript received October 6, 2010; revised January 12, 2011; accepted March 30, 2011. Date of publication August 1, 2011; date of current version September 28, 2011. This paper was recommended by Associate Editor A. Devred.

The authors are with the European Organization for Nuclear Research, 1211 Geneva, Switzerland (e-mail: nikolai.schwerg@ cern.ch).

Color versions of one or more of the figures in this paper are available online at http://ieeexplore.ieee.org.

Digital Object Identifier 10.1109/TASC.2011.2157344
The requirements on field uniformity in the apertures of the superconducting magnets also posed a challenge to numericalfield-computation and optimization techniques. The computation of the fields in the magnet aperture requires an accuracy of six digits. Yet, the modeling must be flexible and fast in order to allow for optimization routines to determine optimum design parameters. The coupling method of boundary elements and finite elements (BEM-FEM) corresponds to both requirements as the magnet apertures are contained in the BEM regions. The coil fields can be computed to machine precision from the Biot-Savart law. Only the nonlinear iron yoke needs to be modeled by a finite element mesh [3].

The CERN field-computation program ROXIE [4] has been developed by combining the BEM-FEM technique, parametric coil modeling and meshing, and mathematical optimization techniques. Models for transient-field effects in superconducting cables were also implemented. It is possible to calculate the effect of persistent currents, interfilament-coupling currents (IFCCs), and interstrand-coupling currents (ISCCs) on the field quality [5]. Furthermore, these models provide the input in terms of thermal losses during a nominal ramp cycle and a transition to the magnet's normal conducting state (quench). The ROXIE program was used equally to trace manufacturing errors by inverse field computation.

After an ambitious hardware-commissioning phase, a first beam was circulated on September 10, 2008. In this paper, we review the role that numerical field computation plays during the hardware-commissioning phase, i.e., the period when all circuits of magnets in the LHC tunnel were tested and their behavior was validated.

\section{3-D Force AND Peak-Field CAlculations}

Electromagnetic forces on interconnection busbars were identified during commissioning to be an important issue for the long-term reliability and the electromechanical integrity of the machine. While field-quality calculations for long accelerator magnets can be carried out to highest precision in 2-D calculations, the interconnect regions between magnet coils and neighboring magnets require a 3-D approach. Applying the BEM-FEM technique, the FEM modeling could be restricted to the nonlinear ferromagnetic yoke. The involved layout of cosine-theta-type coil ends and the busbar routing was described in a Biot-Savart-type model built from straight linecurrent segments.

The Biot-Savart model was generated from basic building blocks such as easy- and hard-way bend and twists. The twist pitch of the Rutherford-type cable is neglected. An automatic 


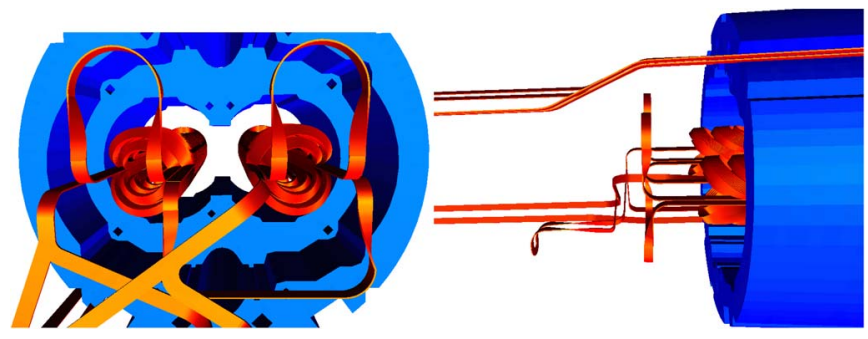

Fig. 1. Electromagnetic model of the interconnections at the end regions of the main bending dipoles of the LHC.

connection routine computes the transformations that are required for a continuous interconnection of individual components. The complete BEM-FEM model consists of about 600000 line-current segments in the BEM domain and only about 60000 finite elements. The calculation of forces on the interconnection busbars takes approximately $2 \mathrm{~h}$ on a $2.8-\mathrm{GHz}$ Xeon processor machine.

Despite the relatively low number of finite elements in the model, the accuracy of peak-field calculations on the superconducting cable exceeds by far the precision that could be achieved in a pure finite elements model. The reason is that the local field distribution in the cables is determined by the current flow in individual strands, which cannot be represented adequately in a FEM approach. We note that the forces pulling in the longitudinal direction (along the magnet axis) on the so-called half-moon interconnects (the $180^{\circ}$ arcs above both apertures) [see Fig. 1 (left)] that connect individual coils in a magnet assembly are on an average of $240 \mathrm{~N}$.

\section{QuenCH Simulation}

\section{A. Active And Passive Quench Protection}

Above a certain limit on temperature, current density, and magnetic-flux density, superconductors show a transition between the superconducting and normal conducting states. The range of parameters that marks a transition is called the critical surface. A quench is a transition that causes an amount of resistive losses, which cannot be absorbed by the cooling system. Quench detection and magnet protection against overheating and voltage peaks during a quench are important issues in the design of superconducting magnets [6]. An incipient quench is detected by the resistive-voltage rise across the normal zone, which must be distinguished from the induced voltage during the ramping of the magnet. This is accomplished by a bridgedetection system. Magnet-protection schemes can be classified in two groups. Passive-protection schemes may include a diode or a resistor connected in parallel to the magnet, but principally, they rely on strong stabilization of the conductor, such that the magnet can withstand the current decay without overheating. A stabilized conductor is a conductor with a copper-tosuperconductor ratio that is large enough so that, in case the superconductor quenches, the copper can take over the current for a long enough time to ramp down the magnet safely [6].

We speak of active protection when measures are taken to speed up the normal-zone propagation and the current decay in the magnet. The current decay is determined by the propagation speed of the normal zone and by the external electrical circuit connected to the magnet. Due to the high inductance of superconducting-magnet circuits, the current cannot be switched off instantaneously; therefore, the power supply is short circuited with a freewheeling diode. The currentdecay rate is given by the inductance and the resistance of the remaining circuit.

Active protection relies on quench heaters and/or on an energy-extraction system. We will be concerned with protection by heaters. The heaters cause a resistive transition in the covered coil windings, ensuring that the stored energy is dissipated over a larger fraction of the coil volume. The rising resistance decreases the discharge time constant and thus reduces the hot-spot temperature. A quench-heater circuit consists of a stainless-steel heater strip colaminated with polyimide insulation, a thyristor, and an aluminum electrolytic capacitor bank. Upon trigger, the capacitor bank is discharged across the resistance of the heater strip.

As the normal zone propagates along the superconducting cable, the coil-winding scheme of the magnet with its internal and external connections must be taken into account. The electrical connection of the twin-aperture LHC main dipole is shown in Fig. 2. The current entering the terminal A of the magnet will flow first through the lower outer-layer coil of the inner aperture. The inner aperture is the one at the righthand side of an observer looking downstream of the circulating Beam 1. The current will then enter the lower inner, upper inner, and upper outer coils before it will be directed to the outer aperture. Fig. 2 also shows the quench-heater circuits and the outer-layer cables covered by the heaters. Only the high-field heaters (circuits 211, 221, 111, and 121) are powered usually, and the low-field heaters are mounted for redundancy. ${ }^{1}$

The typical values for the protection system of the LHC main dipole are as follows. The threshold voltage is $0.1 \mathrm{~V}$; the quench heaters are triggered after a delay of $10 \mathrm{~ms}$ needed for signal validation [7]. The time constant for the dissipated heater power is $37 \mathrm{~ms}$; measurements indicate that a heater-provoked quench at $1.5 \mathrm{kA}$ occurs at around $80 \mathrm{~ms}$ after the trigger. At nominal current, the delay reduces to $35 \mathrm{~ms}$ [8].

\section{B. Modeling Quench}

The field distribution in the coil is calculated by means of the coupling method between boundary and finite elements (BEM-FEM). For accelerator magnets, the numerical field computation can be often restricted to two dimensions. The rapid current decay during a quench creates losses due to the IFCCs and the ISCCs. The coupling-current time constants are influenced by the copper resistivity, the contact resistances, and geometric cable parameters; all of which are input parameters to the simulation.

In the superconducting state, the working point lies below the critical surface. At every time step, the temperature margin of the cable to the critical surface is evaluated as a function

\footnotetext{
${ }^{1}$ The naming convention for the quench-heater circuits is as follows: XYZ, where $\mathrm{X}$ is the aperture ( 1 or 2 ), $\mathrm{Y}$ is the position at the coil (1 for left and 2 for right), and $\mathrm{Z}$ stands for the low- or high-field region (1 for high and 2 for low).
} 


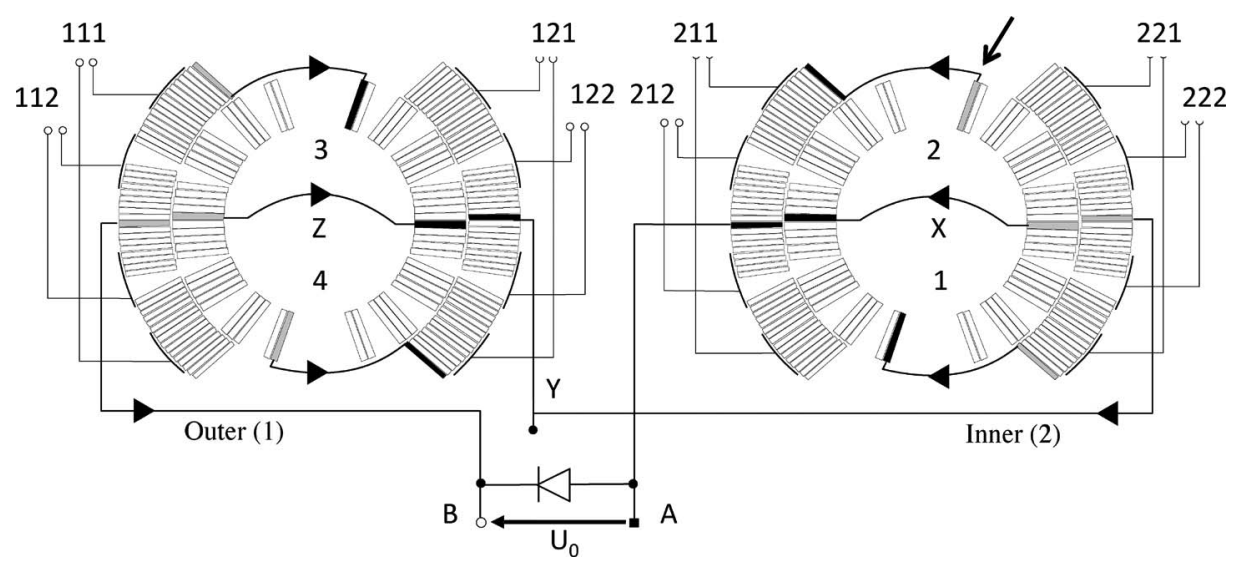

Fig. 2. Winding scheme and internal connections in the double-aperture LHC main-dipole magnet together with the position of quench heaters. The arrow indicates the turn where the quench is triggered in our simulations.

of the peak field and the current density. The copper resistivity is calculated as a function of the temperature and the average magnetic-flux density in the cable cross section. The average magnetic-flux density is taken because of the transposition of the strands within the cable and the 2-D approximation of the field problem. The dissipated power in each cable is the sum of resistive losses due to the current in the copper matrix of the normal zone, losses due to coupling currents (IFCCs and ISCCs), the heat transferred from the quench heaters, and beam losses. The coupling-current losses can be neglected once the cable has quenched. Quench heaters are characterized by the maximum heater power, a delay of heat transfer, and the time constant of the exponential decay. These parameters can be used to validate the model with the measured thermal coupling between the heaters and the coil [9].

The simulation of thermal processes at cryogenic temperatures is an intricate problem. Material properties at cryogenic temperatures and under pressure are often difficult to know to adequate precision. The highly nonlinear behavior of these parameters leads to an ill-conditioned numerical problem in quench simulations. Moreover, the problem is generally ill posed as there are more model parameters than validation criteria [10] such as measurements of the current decay and signals at the voltage taps or the quench antenna.

The electromagnetic and thermal models exhibit different time constants. Moreover, field calculation is computationally more expensive than the solution of the coupled electrical and thermal network equations. Hence, a weak coupling between the electromagnetic and thermal models is the most efficient method to solve the multiphysics problem. The integrated numerical model allows to study the impact of different effects such as quench-back, normal zone propagation, quench-heater performance, local field distribution, and iron saturation [9].

\section{Quench Simulation for Hardware Commissioning}

In the commissioning phase of the LHC, a quench-heater connection broke after the cooldown of a dipole magnet to the operation temperature [11]. As a replacement of a magnet in the string of superconducting magnets is very costly and time consuming, the low-field quench heaters, which were built-in
TABLE I

QUENCH-HeATER-POWERING SCHEMES; COMPARE FIG. 2

\begin{tabular}{l|l}
\hline \hline Case & Heater configuration \\
\hline 1 & No heaters \\
2 & $(112+122), 121,211,(212+222)$ \\
3 & $111,121,211,221($ LHC operation conditions) \\
4 & $111,121,211,222$ \\
5 & $111,121,212,222$ \\
6 & $112,121,211,222$ \\
7 & $112,122,212,222$ \\
8 & $111,121,211,(212+222)$ \\
\hline
\end{tabular}

for redundancy, were to replace the damaged specimen. By means of quench simulation, an optimum powering scheme for the remaining quench-heater circuits was to be found. The hot-spot temperature, the maximum voltage to ground, and the maximum electric field in the coil windings during the quench were identified as decisive criteria.

The study consists of eight different heater-powering schemes. Using the heater numbering in Fig. 2, the cases are summarized in Table I. Case 1 represents the hypothetical case in which no quench heaters fire. Case 3 shows the nominal protection scheme. Four power supplies are available to fire the quench heaters. One supply may be used to fire two heaters in parallel. This case is indicated above by brackets. In all simulations, the quench is assumed to originate in the innermost turn of coil 2 (see the arrow in Fig. 2). The damaged heater is assumed to be number 221 .

The model parameters for the quench heaters have been determined in [12]. In the case of two heaters connected in parallel, the time constant of the heat pulse halves. In addition, the energy that is deposited in the conductors, which are covered by the heaters, is reduced by a factor of 2 .

The simulation results are summarized in Table II, which shows the peak temperature, the quench load, the maximum voltage to ground, and the maximum electrical field between adjacent conductors. For Case 1, the simulation was stopped when a temperature of $600 \mathrm{~K}$ was reached; at which point, the magnet is considered to be damaged.

The quench load, i.e., the squared current integrated over time, is a function of the quench current, the quench-heater delay, and the time constant. Low-field heaters have a longer delay time (about $20 \mathrm{~ms}$ longer) as a consequence of the larger 
TABLE II

Peak Temperature, Quench Load, Maximum Voltage to Ground, AND Electric Field in the LHC MAIN Dipole as a FunCtion OF THE QUENCH-HEATER-POWERING SCHEME

\begin{tabular}{l|c|c|c|c}
\hline \hline Case & $T_{\max }$ & Quench load & $U_{\max }$ & $E_{\max }$ \\
\hline & $\mathrm{K}$ & $10^{6} \mathrm{~A}^{2} \mathrm{~s}$ & $\mathrm{~V}$ & $\mathrm{MVm}^{-1}$ \\
\hline 1 & 600 & 64 & 576 & 1.19 \\
2 & 280 & 33 & 296 & 0.44 \\
3 & 244 & 32 & 441 & 0.90 \\
4 & 267 & 33 & 768 & 1.13 \\
5 & 299 & 35 & 1248 & 1.75 \\
6 & 299 & 35 & 273 & 0.62 \\
7 & 440 & 42 & 415 & 0.38 \\
8 & 260 & 34 & 668 & 1.06 \\
\hline \hline
\end{tabular}

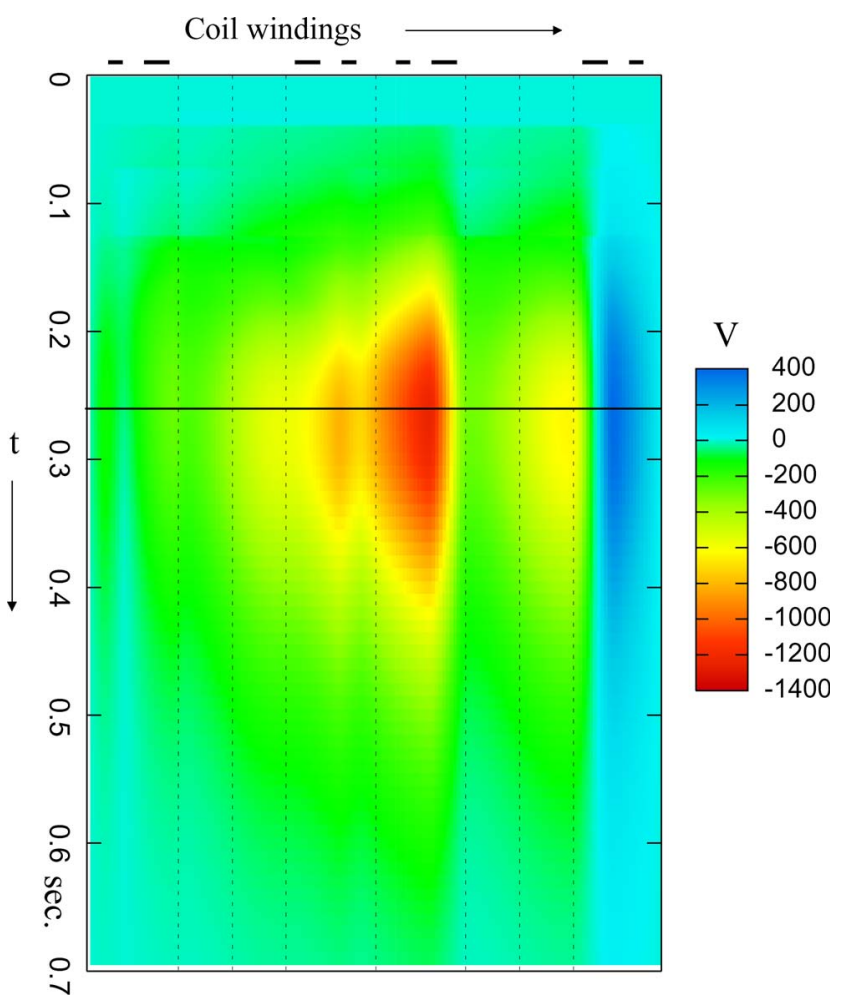

Fig. 3. Electric potential of coil windings as a function of time. Quench-heater powering according to Case 5 .

temperature margin. Moreover, due to magnetoresistive effects, the resistivity of the conductors, which are fired by low-field heaters, is lower than that of conductors covered by high-field heaters. In conclusion, the use of low-field heaters in replacement for high-field heaters increases the quench load, which is reflected in a rising peak temperature. The peak temperature is increased further if hot-spot movement occurs, i.e., if the temperature in conductors covered by quench heaters increases faster than the temperature at the quench origin.

Fig. 3 shows the voltage to ground for each of the coil windings as a function of time. The configuration of Case 5 is depicted, where the high-field quench heaters of the inner aperture (2) are replaced by the low-field heaters.

The voltage to ground as a function of the winding number is displayed for the Cases 2, 3, and 5 at the time of maximum amplitude in Fig. 4. For the simulation, we assume that the terminal $\mathrm{A}$ is connected to ground, whereas terminal B is connected to the cold bypass diode, yielding a static terminal

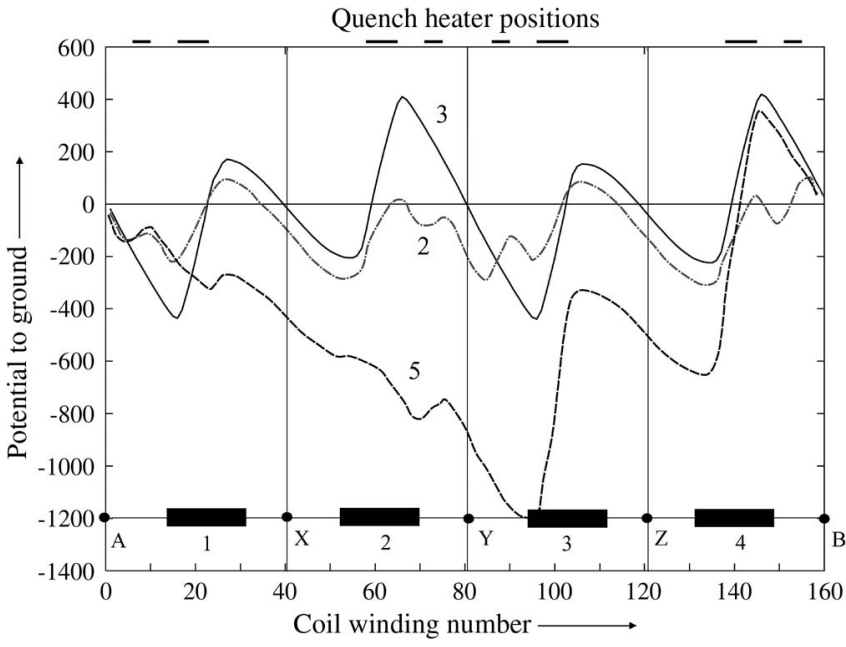

Fig. 4. Electric potential of the coil windings for different powering schemes (Case 2, 3, and 5) of the quench heaters.

voltage of $6 \mathrm{~V}$ for as long as the diode is conductive. A negative slope in the graphs indicates predominantly inductive voltage in the respective windings, whereas a positive slope is due to resistive voltages. Fig. 2 shows the winding scheme and the positions of heaters, voltage taps, and connection terminals.

For the nominal heater setup, i.e., Case 3, we identify easily four positive slopes due to high-field heaters (see Fig. 4). The voltage distribution along the winding is symmetrical with respect to the winding midpoint. While the peak voltage is of the order of several hundred volts, the voltage difference between the two apertures is negligible. In Case 5, one aperture is quenched with high-field heaters, and the other one is quenched with low-field heaters. The strong imbalance in resistive voltage creates a voltage to ground of more than $1.2 \mathrm{kV}$. It can be said that the outer aperture discharges the stored energy in the inneraperture coils, resulting in a greater temperature rise. In Case 2, all low-field heaters and two high-field heaters are employed. This setting ensures a good balance of inductive and resistive voltages over the entire winding, resulting in the lowest voltages in this study.

Eventually, the scheme of Case 8 was applied to replace the damaged heater. In this solution, all remaining high-field heaters are fired, as well as two low-field heaters. The peak voltages are higher than in other schemes. However, the firing of all remaining high-field heaters results in a reduced quench load and an optimized peak temperature.

\section{Field-Quality Simulation}

The operation of the LHC requires that all field errors in the superconducting magnets are compensated for by dedicated corrector magnets so that the integrated field error, as seen by the particle beam, remains below a tolerated limit. Beam-based measurements can serve as feedback on the field quality and as an input for automated controls of the corrector magnets. Yet, for a large-scale machine such as the LHC, operators cannot rely solely on feedback systems. For this reason, the Field Description of the LHC (FiDeL) program collects measurement data of all components in the LHC and extracts a fast online 
model that yields the field quality at a given time, magnet operating current, magnet ramp rate, magnet temperature, and magnet powering history [13]. The modeling is critical particularly at very low currents, where the persistent current magnetization of superconducting strands has a large influence on the field quality. The FiDeL model is based on measurements that were performed for quality assurance during the production of the LHC magnets. Simulation is performed if required for three reasons: 1) to validate the FiDeL mathematical model; 2) to countercheck measurement data; and 3) to supply data where measurements are not available.

The FiDeL model is based on the identification and the physical decomposition of the effects that contribute to the total field in the magnet aperture. Each effect is modeled by an appropriate mathematical model. The physical behavior of the models can be tested over the entire parameter space by means of simulation. With the exception of decay- and snapback effects, the ROXIE software comprises all relevant effects. A database of electromagnetic models of all LHC superconducting magnets is used to compare model and measurement results. Suspicious measurement data can be tested and validated by comparison. In the low-field region, where the persistentcurrent effects on field quality are dominant, the ROXIE model [14] can yield data for powering cycles that have not been measured previously.

\section{CONCLUSION}

Numerical field computation is indispensable in the design and manufacturing phase of accelerator magnets. However, computational challenges arise also from the commissioning phase. Understanding the actual magnet behavior, contributing to risk analysis, helping to improve the overall understanding of the machine, and working on fallback solutions in the case of equipment failure are some of the tasks encountered during the LHC commissioning. To keep the response time to computation requests short, up-to-date numerical models of all equipment must be maintained for all magnets installed in the accelerator tunnel.

\section{REFERENCES}

[1] P. Lefèvre and T. Pettersson, Eds., The Large Hadron ColliderConceptual Design. Geneva, Switzerland: CERN, 1995.

[2] O. Brüning, P. Collier, P. Lebrun, S. Myers, R. Ostojic, J. Poole, and P. Proudlock, Eds., LHC Design Report-The LHC Main Ring. Geneva, Switzerland: CERN, 2004.

[3] S. Kurz, J. Fetzer, and W. M. Rucker, "Coupled BEM-FEM methods for 3D field calculation with iron saturation," in Proc. 1st Int. ROXIE Users Meeting Workshop, Mar. 1998, pp. 47-59.
[4] S. Russenschuck, Field Computation for Accelerator Magnets, 1st ed. New York: Wiley-VCH, 2010.

[5] B. Auchmann, R. de Maria, and S. Russenschuck, "Calculation of timetransient effects with the CERN field computation program ROXIE," IEEE Trans. Appl. Supercond., vol. 18, no. 2, pp. 1569-1572, Jun. 2008.

[6] Y. Iwasa, "Stability and protection of superconducting magnets-A discussion," IEEE Trans. Appl. Supercond., vol. 15, no. 2, pp. 1615-1620, Jun. 2005.

[7] R. Denz, "Electronic systems for the protection of superconducting elements in the LHC," IEEE Trans. Appl. Supercond., vol. 16, no. 2, pp. 1725-1728, Jun. 2006.

[8] P. Pugnat, Measurement of Quench Heater Delays, private communication, Jul. 2007.

[9] N. Schwerg, "Numerical calculation of transient field effects in quenching superconducting magnets," Ph.D. dissertation, Technische Universität Berlin, Berlin, Germany, Feb. 2010.

[10] N. Schwerg, B. Auchmann, and S. Russenschuck, "Challenges in the thermal modeling of quenches with ROXIE," IEEE Trans. Appl. Supercond., vol. 19, no. 3, pp. 1270-1273, Jun. 2009.

[11] R. Denz, K. Dahlerup-Petersen, and K. H. Mess, "Electronic systems for the protection of superconducting devices in the LHC," CERN, Geneva, Switzerland, Tech. Rep. LHC-PROJECT-Report-1142, Aug. 2008.

[12] N. Schwerg, B. Auchmann, and S. Russenschuck, "Validation of a coupled thermal-electromagnetic quench model for accelerator magnets," IEEE Trans. Appl. Supercond., vol. 18, no. 2, pp. 1565-1568, Jun. 2008.

[13] N. Sammut, L. Bottura, and J. Micallef, "Mathematical formulation to predict the harmonics of the superconducting large hadron collider magnets," Phys. Rev. ST Accel. Beams, vol. 9, no. 1, p. 012 402, Jan. 2006.

[14] M. Aleksa, B. Auchmann, S. Russenschuck, and C. Vollinger, "A vector hysteresis model for superconducting filament magnetization in accelerator magnets," IEEE Trans. Magn., vol. 40, no. 2, pp. 864-867, Mar. 2004.

Nikolai Schwerg received the Diploma and Dr.-Ing. degrees in electrical engineering from the Technical University of Berlin, Germany, in 2006 and 2009 , respectively.

Since 2006, he has been with the European Organization for Nuclear Research (CERN), Geneva, Switzerland, at first on the electromagnetic simulation of superconducting magnets and currently as a Senior Fellow on the design and the simulation of high-power radio-frequency systems.

Bernhard Auchmann was born in Vienna, Austria, in 1979. He received the Diploma and Ph.D. degrees in electrical engineering from the Vienna University of Technology, Vienna, Austria, in 2001 and 2004, respectively.

Since 2002, he has been with the European Organization for Nuclear Research (CERN), Geneva, Switzerland, where he is currently a Scientific Staff on the electromagnetic simulation of superconducting magnets.

Stephan Russenschuck received the Dr.-Ing. degree in electrical engineering from the Technische Universität Darmstadt, Darmstadt, Germany, in 1990.

In 2000, he was recognized as a University Lecturer on the theory of electromagnetic fields with the University of Vienna, Austria. He is currently a Senior Staff Member with the European Organization for Nuclear Research (CERN), Geneva, Switzerland, where he leads the magnetic-field measurement activity with the Technology Department. He is the author of the CERN-ROXIE program, i.e., an integrated design software for accelerator magnets, and the textbook "Field Computation of Accelerator Magnets," published by Wiley$\mathrm{VCH}$. His research interests include mathematical optimization and numerical field computation techniques for magnet design. 